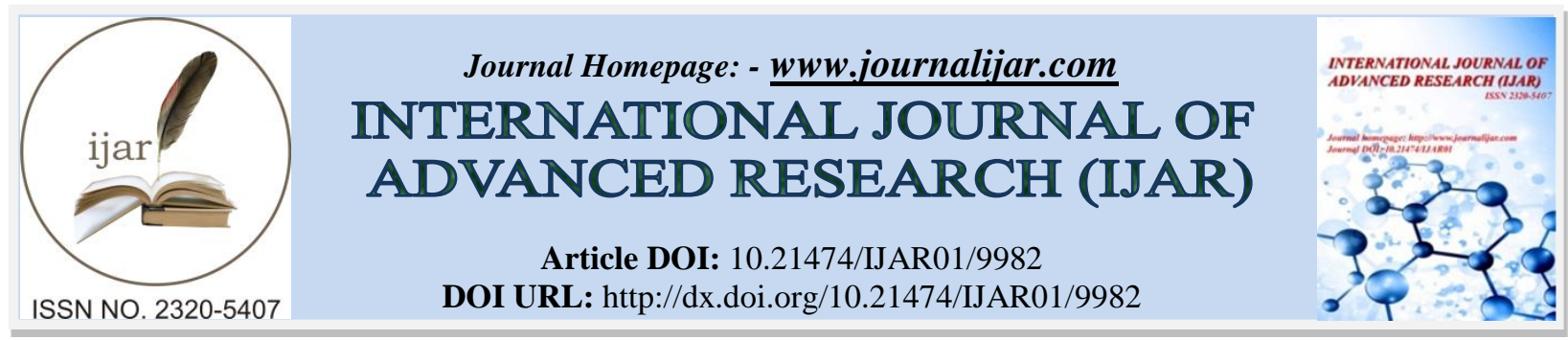

RESEARCH ARTICLE

\title{
NUMERICAL ANALYSIS OF SINGLE AND TWO RELAY COOPERATIVE COMMUNICATION SYSTEM WITH DIFFERENT DIVERSITY PROTOCOLS.
}

Md. Mahabub Hossain, Md. Dulal Haque and Md. Mehedi Islam.

Department of Electronics and Communication Engineering, Hajee Mohammad Danesh Science and Technology University, Dinajpur-5200, Bangladesh.

\section{Manuscript Info}

Manuscript History

Received: 05 September 2019

Final Accepted: 07 October 2019

Published: November 2019

\section{Key words:-}

Wireless networks, cooperative diversity, relay, diversity protocols and combining methods.

\section{Abstract}

In this paper the comparative study of a cooperative communication system is analyzed under single and two-relay as well as different diversity protocol. The single and two relays with two retransmission protocols amplify and forward (AAF) and decode and forward (DAF) are implemented as well as four diversity combining techniques such as equal ratio combining (ERC), fixed ratio combining (FRC), signal to noise ratio combining (SNRC) and equal signal to noise ratio combining (ESNRC) are used at the destination for each protocol. When ERC or FRC is implemented for both AAF and DAF diversity protocols the two-relay system presents better performance compared to the single relay. On the other hand, two-relay cooperative system has shown improved system performance at lower SNRs for SNRC or ESNRC. The proposed two relays system using cooperative diversity can achieve remarkable benefits in wireless transmission and the performance can be enhanced of the transferred signal with improved channel quality.

Copy Right, IJAR, 2019,. All rights reserved.

\section{Introduction:-}

The signal quality degrades due to bad channel quality for the effect of fading caused by multi-path propagation loss during the transmission. For the reduction of the such effects the diversity technique can be implemented to transmit or receive the independent samples of the signal along different propagation paths using multiple antenna. But the wireless terminals might not be able to support multiple antennas owing to hardware limitations [1, 2]]. To overcome these problems, a new diversity technique cooperative relaying diversity can also be realized by using single antenna in a multi-user environment and generate a virtual multiple-antenna transmitter that allows them to achieve transmit diversity. The relay virtually performs as another transmitter and enables the source to communicate with the destination [3]. The two independent wireless links transmit the information, one used as a direct link and another is the multi-hop link which results in diversity gain. The selection of the path at destination with the best signal quality as well as processing and forwarding of the incoming signals by the relaysare some important issues. The investigation for path loss has been perform for different relays from a minimum energy perspective [4, 5]. J. Boyer et al. [5] has proposed a cooperative system where the intermediate relays recombine, digitally decode and re-encode or simply combine and amplify the received signals from all preceding terminals. For one relay cooperative system, Amplify and Forward (AAF) and Decode and Forward (DAF) cooperative relaying protocols achieve full diversity [6-9]. Moreover, Amplify and Forward relaying outperforms both direct

Corresponding Author: - Md. Dulal Haque.

Address: - Department of Electronics and Communication Engineering, Hajee Mohammad Danesh 
transmission and Decode and Forward relaying despite noise propagation [10-12]. For realization of robust communication system, it is very much essential to analyze the effect single and multiple relays for cooperative communication channel to achieve diversity gain.

The fundamental purpose of our investigation is to analyze the single and two relays schemes for cooperative communication. We have performed numerical analysis for the single and two relays cooperative relaying with AAF and DAF schemes. Finally, the results of single and two relays schemes have been compared for enhancing the diversity gain. The single and two-relay cooperative communication systems are shown in Figs. 1 and 2, respectively.

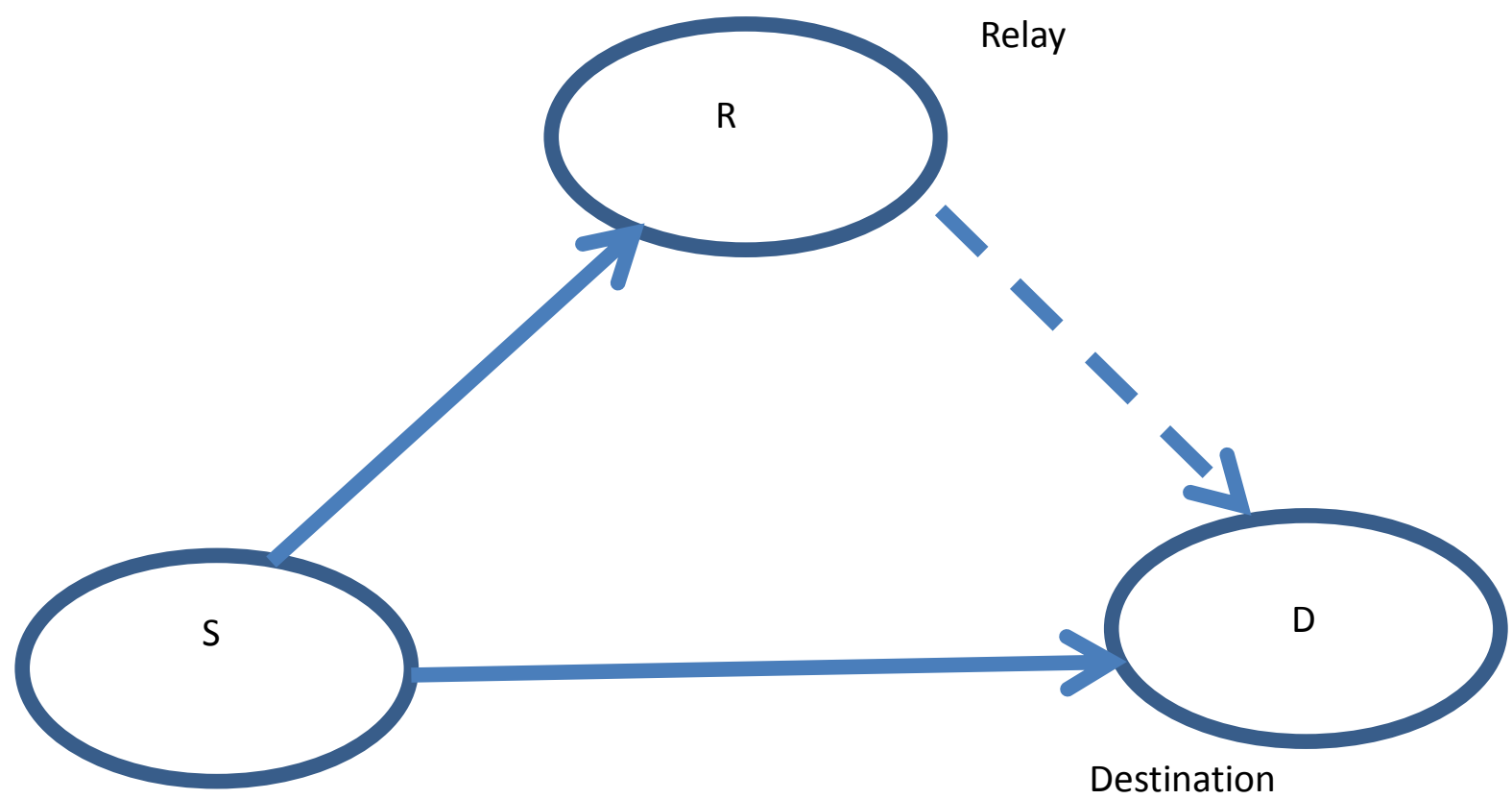

Source

Fig 1:- A single relay cooperative communication system model.

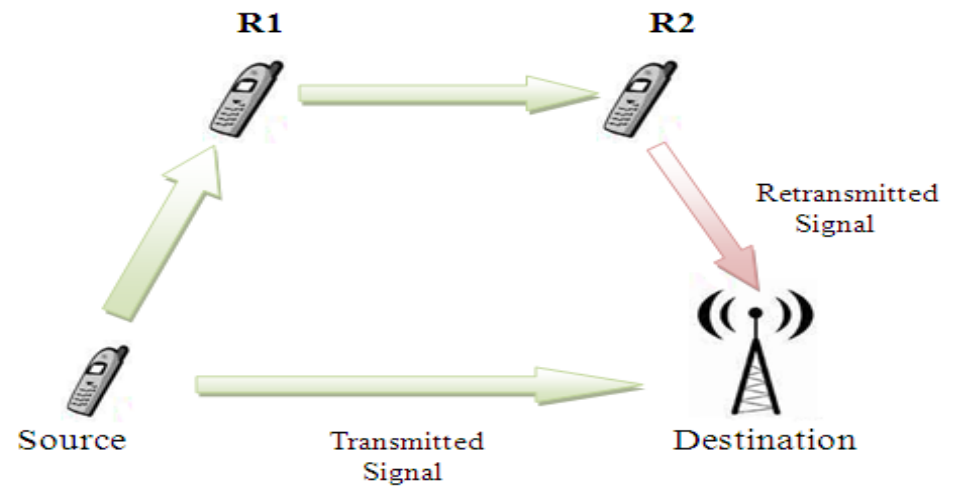

Fig 2:- A two-relay cooperative communication system model.

\section{Diversity Protocols:}

The complete system that is considered for analysis includes a source, two serial relays- relay1 (R1) and relay2 (R2), and a destination as shown in Fig. 2. The channel between source and destination, (source and R1), (R1 and R2) and (R2 and destination) are modeled as independent rayleigh fading channels. Here source can be considered as a 
conventional transmitter; both relays as transceivers with AAF and DAF transmission protocols, and destination as a receiver containing the combining function. For simplicity, we consider a half-duplex system model in which two versions of the original signal is transmitted through two different paths of same path length. One is transmitted directly from the source to the destination through single link and another is transmitted through the multi-hop.

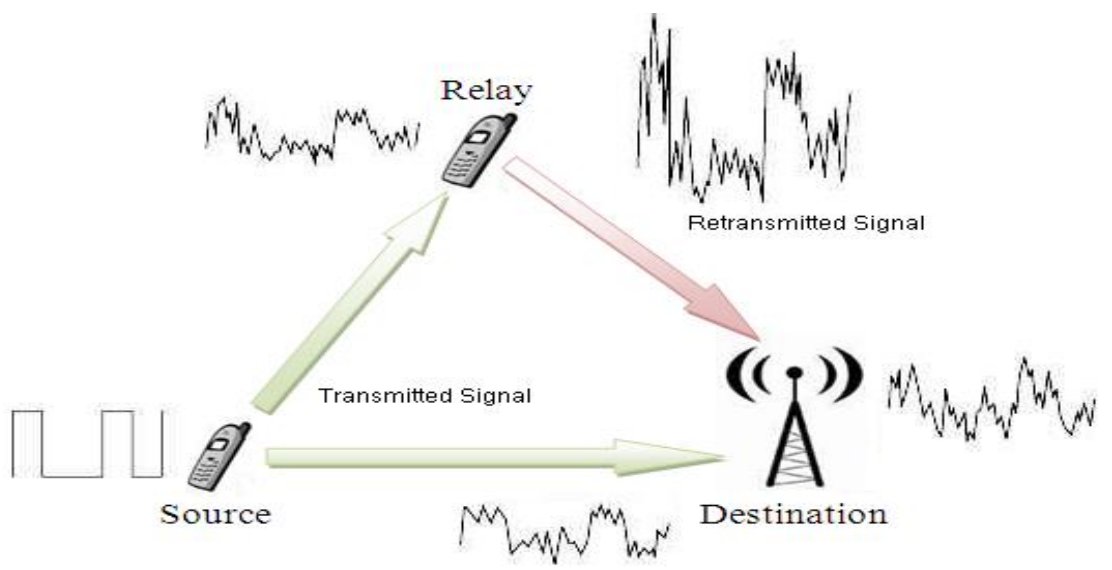

Fig 3:-Amplify and Forward.

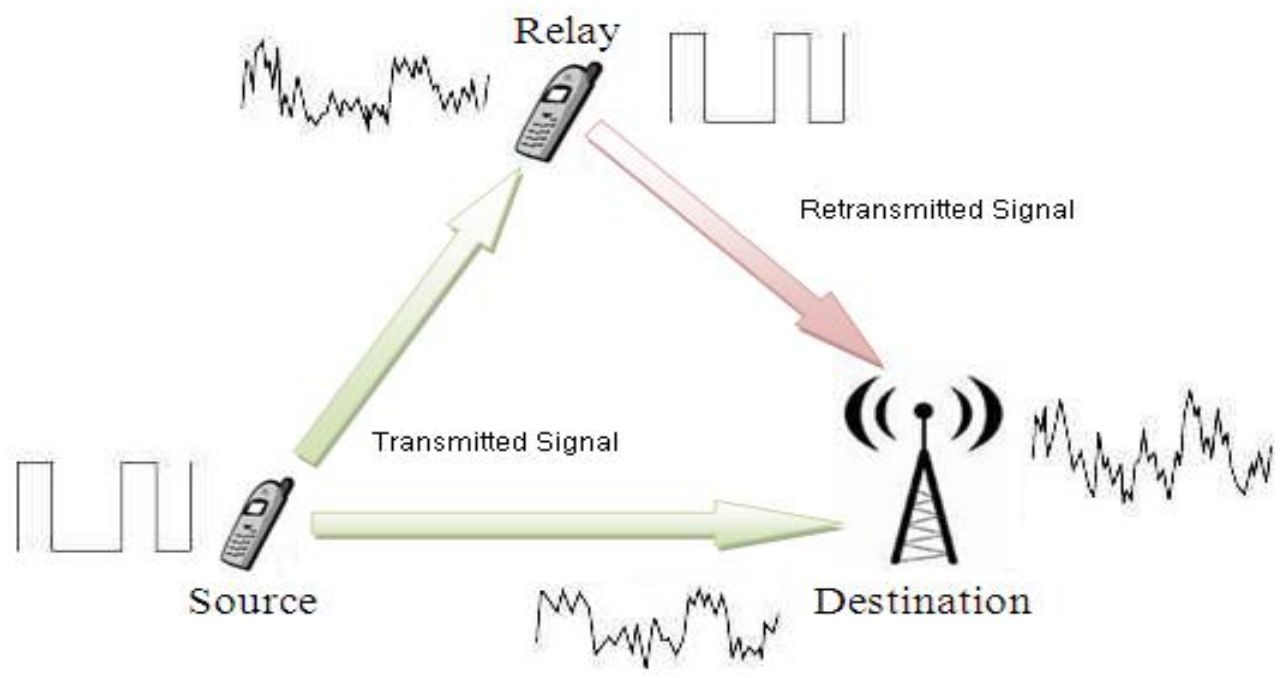

Fig 4:- Decode and Forward

The idea behind the AAF protocol, which was was proposed and analyzed by Lanemanet al. which is simple shown in Fig. 3. Each relay receives a noisy version of the transmitted signal and then amplifies and retransmits it [13-15]. In doing so the noise in the signal is amplified as well, this is the main downfall of this protocol. Though noise is amplified by cooperation, the destination can make better decision on the transmitted bits by combining the two independently received faded versions of the signals.

On the other hand, DAF shown in Fig. 4 is most often the preferred method to process the data in the relay [15-16]. The received signal is first decoded and then re-encoded. So there is no amplified noise in the sent signal, as is the case using a AAF protocol. The relay can decode the original message completely. This requires a lot of computing time, but has numerous advantages. If the source message contains an error correcting code, received bit errors might be corrected at the relay station [15]. Or if there is no such code implemented a checksum allows the relay to detect if the received signal contains errors. But it is not always possible to fully decode the source message. The additional delay caused to fully decode and process the message is not acceptable, the relay might not have enough computing capacity or the source message could be coded to protect sensitive data. In such a case, the incoming 
signal is just decoded and re-encoded symbol by symbol. So neither an error correction can be performed nor a checksum calculated.

\section{Numerical model for two relay cooperative System with AAF and DAF protocols}

The estimation of the SNR of a multi-hop link using AAF or a direct link can be performed by sending a known symbol sequence in every block. If the multi-hop link is using a DAF protocol, the receiver can only see the channel quality of the last hop. It is assumed that the relay sends some additional information about the quality of the unseen hops to the destination, so the SNR of the multi-hop link can be estimated as well. Whatever protocol is used, an additional sequence needs to be sent to estimate the channel quality. This results a certain loss in bandwidth. The model is represented in Fig. 5.

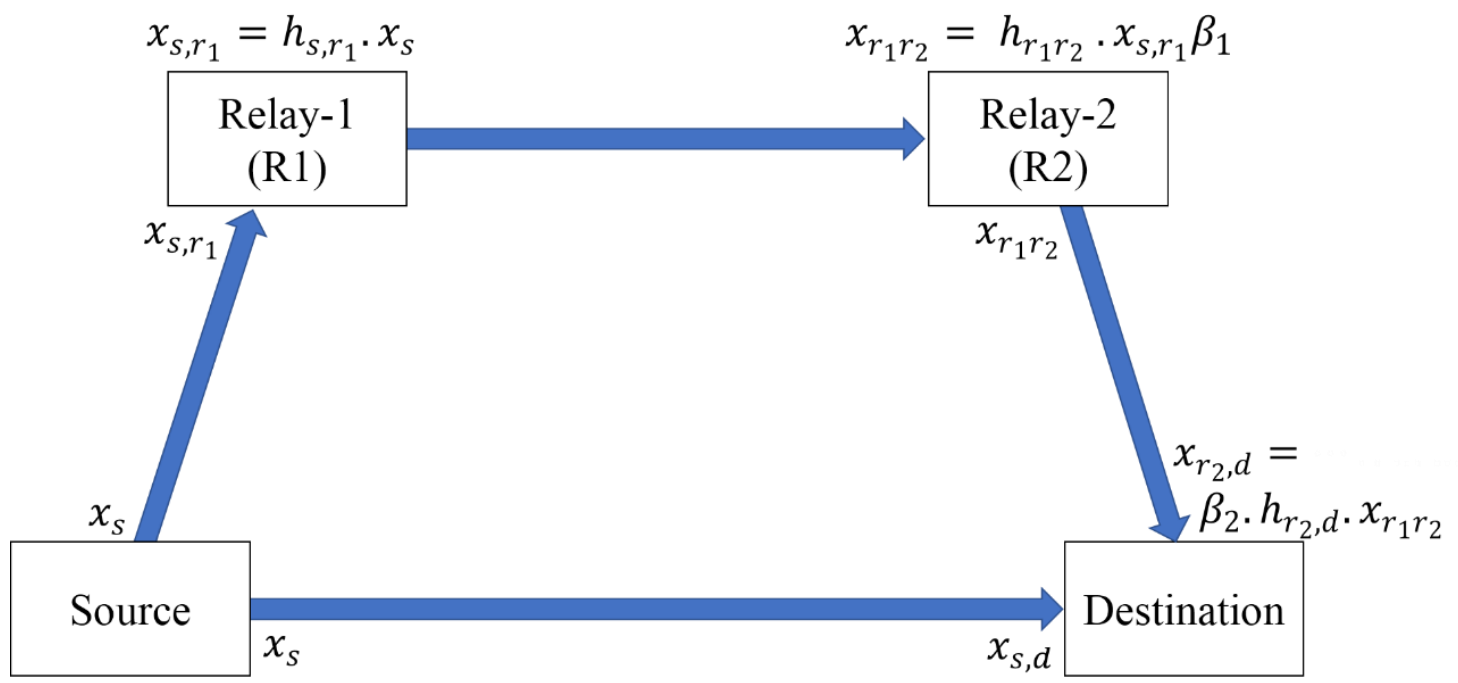

Fig 5:- Cooperative system model for two serial relays

\section{Estimation of SNR using AAF}

In this case, the relay R1 simply amplifies the received signal from source and transmits it to relay R2. Then R2 again amplifies the received signal and retransmits it to destination.

The received signal at the relay $\mathrm{R} 1$ is,

$\mathbf{x}_{\mathrm{s}, \mathbf{r}_{1}}=\underbrace{\mathbf{h}_{\mathbf{s}, \mathbf{r}_{1}}}_{\text {attenuation }} \cdot \mathbf{x}_{\mathbf{s}}+\underbrace{\mathbf{z}_{\mathrm{s}, \mathbf{r}_{1}}}_{\text {noise }}$

Where $\mathrm{x}_{\mathrm{s}}$ is transmitted signal by the source. Path loss and fading are included in $\mathrm{h}_{\mathrm{s}, \mathrm{r}_{1}}$.

Power of the incoming signal at relay $\mathrm{R} 1$ is,

$\mathbf{E}\left[\left|\mathbf{x}_{s, \mathbf{r}_{1}}\right|^{2}\right]=\mathbf{E}\left[\left|\mathbf{h}_{s, r_{1}}\right|^{2}\right] \cdot \mathbf{E}\left[\left|\mathbf{x}_{s,}\right|^{2}\right]+\mathbf{E}\left[\left|\mathbf{z}_{s, r_{1}}\right|^{2}\right]$

$\mathbf{E}\left[\left|\mathbf{x}_{\mathrm{s}, \mathbf{r}_{1}}\right|^{2}\right]=\left|\mathbf{h}_{\mathrm{s}, \mathbf{r}_{1}}\right|^{2} \cdot \xi+\underbrace{2 \sigma_{s, \mathbf{r}_{1}}^{2}}_{\text {noise variance }}$

Where $\boldsymbol{\xi}=\mathbf{E}\left[\left|\mathbf{x}_{\mathbf{s}}\right|^{2}\right]$ denotes the energy of the source signal. The source and both relays are assumed to have equal energy per bit $\xi$ and equal noise variance.

Signal to Noise Ratio at relay R1 is,

$\operatorname{SNR}=\frac{\left|\mathbf{h}_{\mathbf{s}, \mathbf{r}_{1}}\right|^{2} \xi}{2 \sigma_{s, \mathbf{r}_{1}}^{2}}$

Signal received by the relay $\mathrm{R} 2$ is, 
$\mathbf{x}_{\mathbf{r}_{1} \mathbf{r}_{2}}=\mathbf{h}_{\mathbf{r}_{1} \mathbf{r}_{2}} \cdot \mathbf{x}_{s_{\mathbf{s}} \mathbf{r}_{1}} \boldsymbol{\beta}_{1}+\mathbf{z}_{\mathbf{r}_{1} \mathbf{r}_{2}}$

$\mathbf{x}_{\mathrm{r}_{1} \mathbf{r}_{2}}=\boldsymbol{\beta}_{1} \mathbf{h}_{\mathbf{r}_{1} \mathbf{r}_{2}}\left(\mathbf{h}_{\mathbf{s}, \mathbf{r}_{1}} \mathbf{x}_{\mathbf{s}}+\mathbf{z}_{s, \mathbf{r}_{1}}\right)+\mathbf{z}_{\mathbf{r}_{1} \mathbf{r}_{2}}$

Where $\boldsymbol{\beta}_{\mathbf{1}}=\sqrt{\frac{\xi}{\left|\mathbf{h}_{\mathbf{s}, \mathbf{r}_{1}}\right|^{2} \xi+2 \sigma_{\mathbf{s}, \mathbf{r}_{\mathbf{1}}}^{2}}}$ is the amplification factor/gain of relay R1.

Power of the incoming signal at relay $\mathrm{R} 2$ is,

$\mathbf{E}\left[\left|\mathbf{x}_{\mathrm{r}_{1} \mathbf{r}_{2}}\right|^{2}\right]=\boldsymbol{\beta}_{1}^{2} \cdot \mathbf{E}\left[\left|\mathbf{h}_{\mathrm{r}_{1} \mathbf{r}_{2}}\right|^{2}\right]\left\{\mathbf{E}\left[\left|\mathbf{h}_{s, \mathbf{r}_{1}}\right|^{2}\right] \cdot \mathbf{E}\left[\left|\mathbf{x}_{\mathrm{s},}\right|^{2}\right]+\mathbf{E}\left[\left|\mathbf{z}_{s, \mathbf{r}_{1}}\right|^{2}\right]\right\}+\mathbf{E}\left[\left|\mathbf{z}_{\mathrm{r}_{1}, \mathbf{r}_{2}}\right|^{2}\right]$

$E\left[\left|x_{r_{1} r_{2}}\right|^{2}\right]=\beta_{1}^{2} \cdot\left|h_{r_{1} r_{2}}\right|^{2}\left\{\left|h_{s, r_{1}}\right|^{2} \cdot \xi+2 \sigma_{s, r_{1}}^{2}\right\}+2 \sigma_{r_{1} r_{2}}^{2}$

$$
E\left[\left|x_{r_{1} r_{2}}\right|^{2}\right]=\beta_{1}^{2} \cdot\left|h_{r_{1} r_{2}}\right|^{2} \cdot\left|h_{s, r_{1}}\right|^{2} \cdot \xi+\beta_{1}^{2}\left|h_{r_{1} r_{2}}\right|^{2} \cdot 2 \sigma_{s, r_{1}}^{2}+2 \sigma_{r_{1} r_{2}}^{2}
$$

Signal to Noise Ratio at relay2 is,

$\operatorname{SNR}=\frac{\beta_{1}^{2}\left|\mathbf{h}_{\mathrm{r}_{1} \mathbf{r}_{2}}\right|^{2} \cdot\left|\mathbf{h}_{\mathrm{s}, \mathrm{r}_{1}}\right|^{2} \xi}{\beta_{1}^{2}\left|\mathrm{~h}_{\mathrm{r}_{1} \mathrm{r}_{2}}\right|^{2} \cdot 2 \sigma_{\mathrm{s}, \mathrm{r}_{1}}^{2}+2 \sigma_{\mathrm{r}_{1} \mathrm{r}_{2}}^{2}}$

Received signal at destination from relay $\mathrm{R} 2$ is,

$\mathbf{x}_{\mathbf{r}_{2}, \mathbf{d}}=\boldsymbol{\beta}_{2} \cdot \mathbf{h}_{\mathbf{r}_{2}, \mathbf{d}} \cdot \mathbf{x}_{\mathbf{r}_{1} \mathbf{r}_{2}}+\mathbf{z}_{\mathbf{r}_{2, d}}$

$\mathbf{x}_{\mathrm{r}_{2}, \mathrm{~d}}=\boldsymbol{\beta}_{1} \boldsymbol{\beta}_{2} \cdot \mathbf{h}_{\mathrm{r}_{2}, \mathrm{~d}} \cdot \mathbf{h}_{\mathrm{r}_{1} \mathbf{r}_{2}}\left(\mathbf{h}_{\mathrm{s}, \mathbf{r}_{1}} \cdot \mathbf{x}_{\mathrm{s}}+\mathbf{z}_{\mathrm{s}, \mathbf{r}_{1}}\right)+\boldsymbol{\beta}_{2} \cdot \mathbf{h}_{\mathbf{r}_{2}, \mathrm{~d}} \cdot \mathbf{z}_{\mathbf{r}_{1} \mathbf{r}_{2}}+\mathbf{z}_{\mathrm{r}_{2}, \mathrm{~d}}$

Where $\boldsymbol{\beta}_{2}=\boldsymbol{\beta}_{1}=\sqrt{\frac{\xi}{\left|\mathbf{h}_{\mathbf{r}_{1}, \mathbf{r}_{2}}\right|^{2} \xi+2 \sigma_{\mathbf{r}_{1} \mathbf{r}_{2}}^{2}}}$ is the amplification factor/gain of relay 2. Same

channel effects are assumed for every relay channel. Power of the incoming signal at destination is,

$$
\begin{aligned}
& \mathbf{E}\left[\left|\mathbf{x}_{\mathrm{r}_{2}, \mathbf{d}}\right|^{2}\right]=\boldsymbol{\beta}_{1}^{2} \boldsymbol{\beta}_{2}^{2} \mathbf{E}\left[\left|\mathbf{h}_{\mathbf{r}_{2}, \mathrm{~d}}\right|^{2}\right] \mathbf{E}\left[\left|\mathbf{h}_{\mathrm{r}_{1} \mathbf{r}_{2}}\right|^{2}\right]\left\{\mathbf{E}\left[\left|\mathbf{h}_{\mathrm{s}, \mathbf{r}_{1}}\right|^{2}\right] \mathbf{E}\left[\left|\mathbf{x}_{\mathrm{s},}\right|^{2}\right]+\right. \\
& \left.\mathbf{E}\left[\left|\mathbf{z}_{\mathrm{s}, \mathrm{r}_{1}}\right|^{2}\right]\right\}+\boldsymbol{\beta}_{2}^{2} \mathbf{E}\left[\left|\mathbf{h}_{\mathrm{r}_{2}, \mathrm{~d}}\right|^{2}\right] \mathbf{E}\left[\left|\mathbf{z}_{\mathbf{r}_{1} \mathbf{r}_{2}}\right|^{2}\right]+\mathbf{E}\left[\left|\mathbf{z}_{\mathbf{r}_{2}, \mathrm{~d}}\right|^{2}\right] \\
& \mathbf{E}\left[\left|\mathbf{x}_{\mathrm{r}_{2}, \mathrm{~d}}\right|^{2}\right]=\boldsymbol{\beta}_{1}^{2} \boldsymbol{\beta}_{2}^{2}\left|\mathbf{h}_{\mathrm{r}_{2}, \mathrm{~d}}\right|^{2}\left|\mathbf{h}_{\mathrm{r}_{1} \mathbf{r}_{2}}\right|^{2}\left\{\left|\mathbf{h}_{\mathrm{s}, \mathrm{r}_{1}}\right|^{2} \xi+2 \sigma_{\mathrm{s}, \mathrm{r}_{1}}^{2}\right\}+\boldsymbol{\beta}_{2}^{2}\left|\mathbf{h}_{\mathrm{r}_{2}, \mathrm{~d}}\right|^{2} \cdot 2 \sigma_{\mathrm{r}_{1} \mathbf{r}_{2}}^{2}+2 \sigma_{\mathrm{r}_{2}, \mathrm{~d}}^{2} \\
& E\left[\left|\mathbf{x}_{\mathrm{r}_{2}, \mathrm{~d}}\right|^{2}\right]=\beta_{1}^{2} \beta_{2}^{2}\left|\mathbf{h}_{\mathrm{r}_{2}, \mathrm{~d}}\right|^{2}\left|\mathbf{h}_{\mathrm{r}_{1} \mathrm{r}_{2}}\right|^{2}\left|\mathbf{h}_{\mathrm{s}, \mathrm{r}_{1}}\right|^{2} \xi+\beta_{1}^{2} \beta_{2}^{2}\left|\mathbf{h}_{\mathrm{r}_{2}, \mathrm{~d}}\right|^{2}\left|\mathbf{h}_{\mathrm{r}_{1} \mathrm{r}_{2}}\right|^{2} \cdot 2 \sigma_{\mathrm{s}, \mathrm{r}_{1}}^{2}+\beta_{2}^{2}\left|\mathbf{h}_{\mathrm{r}_{2}, \mathrm{~d}}\right|^{2} \cdot 2 \sigma_{\mathrm{r}_{1} \mathbf{r}_{2}}^{2}+2 \sigma_{\mathrm{r}_{2}, \mathrm{~d}}^{2}
\end{aligned}
$$

Signal to Noise Ratio at destination is,

$\operatorname{SNR}=\frac{\beta_{1}^{2} \beta_{2}^{2}\left|h_{\mathrm{r}_{2}, \mathrm{~d}}\right|^{2}\left|\mathbf{h}_{\mathrm{r}_{1} \mathrm{r}_{2}}\right|^{2}\left|\mathbf{h}_{\mathrm{s}, \mathrm{r}_{1}}\right|^{2} \xi}{\beta_{1}^{2} \beta_{2}^{2}\left|\mathbf{h}_{\mathrm{r}_{2}, \mathrm{~d}}\right|^{2}\left|\mathbf{h}_{\mathrm{r}_{1} \mathrm{r}_{2}}\right|^{2} \cdot 2 \sigma_{\mathrm{s}, \mathrm{r}_{1}}^{2}+\beta_{2}^{2}\left|\mathbf{h}_{\mathrm{r}_{2}, \mathrm{~d}}\right|^{2} \cdot 2 \sigma_{\mathrm{r}_{1} \mathrm{r}_{2}}^{2}+2 \sigma_{\mathrm{r}_{2}, \mathrm{~d}}^{2}}$

which is the SNR of multi-hop link consisting of two serial relay stations.

\section{Estimation of SNR using DAF}

The BER over a two relay multi-hop link can then be calculated as

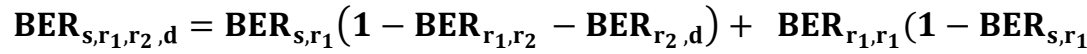

$\left.-\mathrm{BER}_{\mathrm{r}_{2}, \mathrm{~d}}\right)+\mathrm{BER}_{\mathrm{r}_{2}, \mathrm{~d}}\left(1-\mathrm{BER}_{\mathrm{r}_{1}, \mathrm{r}_{2}}-\mathrm{BER}_{\mathrm{s}, \mathrm{r}_{1}}\right)$.

For a BSPK modulated Rayleigh faded signal this will be 
$\operatorname{SNR}=\frac{1}{2}\left[Q^{-1}(B E R)\right]^{2}$

For a QPSK modulated signal this will change toSNR $=\left[\mathbf{Q}^{-\mathbf{1}}(\mathbf{B E R})\right]^{\mathbf{2}}[15-17]$

\section{Diversity Combining Techniques}

The Different diversity combining techniques for one relay have been modified for the application of two serial relays system.

\section{Equal ratio combining (ERC):}

If computing time is a crucial point, or the channel quality could not be estimated, all the received signals can just be added up. This is the easiest way to combine the signals, but the performance will not be that good in return.

$\mathbf{y}_{\mathbf{d}}[\mathbf{n}]=\sum_{\mathbf{i}=\mathbf{0}}^{\mathbf{k}} \mathbf{y}_{\mathbf{i}, \mathbf{d}}[\mathbf{n}]$

Within this thesis two relay stations are used serially as illustrated in Figure 3.1, so the equation simplifies to $\mathbf{y}_{\mathbf{d}}[\mathbf{n}]=\mathbf{y}_{\mathbf{s}, \mathbf{d}}[\mathbf{n}]+\mathbf{y}_{\mathbf{r}_{2}, \mathbf{d}}[\mathbf{n}]$

Wherey $y_{s, d}$ denotes the received signal from the sender and $y_{r_{2}, d}$ denotes the received signal from relay $R 2$.

\section{Fixed ratio combining (FRC):}

The FRC can be expressed as

$\mathbf{y}_{\mathbf{d}}[\mathbf{n}]=\sum_{\mathbf{i}=\mathbf{0}}^{\mathbf{k}} \mathbf{d}_{\mathbf{i}, \mathbf{d}} \cdot \mathbf{y}_{\mathbf{i}, \mathbf{d}}[\mathbf{n}]$

where $d_{i, d} d$ denotes weighting of the incoming signal $y_{i, d}$.

For two serial relay stations, the equation simplifies as

$\mathbf{y}_{\mathrm{d}}[\mathbf{n}]=\mathbf{d}_{\mathrm{s}, \mathrm{d}} \cdot \mathbf{y}_{\mathrm{s}, \mathrm{d}}[\mathbf{n}]+\mathbf{d}_{\mathrm{s}, \mathbf{r}_{1}, \mathbf{r}_{2}, \mathrm{~d}} \cdot \mathbf{y}_{\mathrm{r}_{2}, \mathrm{~d}}[\mathbf{n}]$

where $d_{s, d}$ denotes the weight of the direct link and $d_{s, r_{1}, r_{2}, d}$ denotes the weight of the multi-hop link (Yuksel M and Erkip E 2003).

\section{Signal to noise ratio combining (SNRC):}

The SNRCcan be expressed as

$\mathbf{y}_{\mathrm{d}}[\mathrm{n}]=\sum_{\mathrm{i}=\mathbf{0}}^{\mathrm{k}} \mathrm{SNR}_{\mathrm{i}} \cdot \mathbf{y}_{\mathrm{i}, \mathrm{d}}[\mathrm{n}]$

For two serial relay stations, the equation simplifies as follows

$\mathbf{y}_{\mathbf{d}}[\mathbf{n}]=\operatorname{SNR}_{\mathrm{s}, \mathrm{d}} \cdot \mathbf{y}_{\mathrm{s}, \mathrm{d}}[\mathrm{n}]+\mathrm{SNR}_{\mathrm{s}, \mathbf{r}_{1}, \mathbf{r}_{2}, \mathrm{~d}} \cdot \mathbf{y}_{\mathbf{r}_{2}, \mathrm{~d}}[\mathrm{n}]$

Where $\mathrm{SNR}_{\mathrm{s}, \mathrm{d}}$ denotes the SNR of the direct link and $\mathrm{SNR}_{\mathrm{s}, \mathrm{r}_{1}, \mathrm{r}_{2}, \mathrm{~d}}$ denotes the SNR of the multi-hop link.

\section{Enhanced signal to noise ratio combining (ESNRC):}

For two serial relay stations ESNRC can be expressed as follows, (

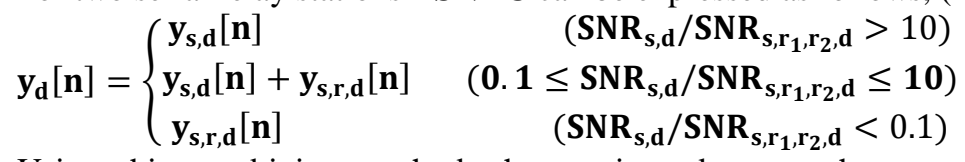

Using this combining method, the receiver does not have to know the channel characteristic exactly. An approximation of the channel quality is enough to combine the signals. As a further benefit, the equal ratio combining does not need a lot of computing power.

\section{Maximum ratio combining (MRC):}

The easiest way to combine the signals in Maximum Ratio Combiner (MRC) is,

$\mathbf{y}_{\mathbf{d}}[\mathbf{n}]=\sum_{\mathbf{i}=\mathbf{1}}^{\mathbf{k}} \mathbf{h}_{\mathbf{i}, \mathbf{d}}^{*}[\mathbf{n}] \cdot \mathbf{y}_{\mathbf{i}, \mathbf{d}}[\mathbf{n}]$ 
For two serial relay stations, the equation simplifies as,

$\mathbf{y}_{\mathbf{d}}[\mathbf{n}]=\mathbf{h}_{\mathbf{s}, \mathbf{d}}^{*}[\mathbf{n}] \mathbf{y}_{\mathbf{s}, \mathbf{d}}[\mathbf{n}]+\mathbf{h}_{\mathbf{r}_{2, d}}^{*}[\mathbf{n}] \mathbf{y}_{\mathbf{r}_{2, d}}[\mathbf{n}]$

\section{Results: -}

\section{Performance analysis of two relay cooperative system}

In Figure 6.1 and 6.2, the performances of two relay cooperative system for different combining methods employing $\mathrm{AAF}$ and DAF forwarding strategies are presented. Two relay cooperative system for different combining techniques using AAF diversity protocol shows better performance compared to single link transmission. SNRC and ESNRC provide almost same performance for the entire SNR. ERC achieves better performance up to $5.65 \mathrm{~dB}$. After that point FRC is the best at higher SNR. In this case as like as AAF, DAF in fig. 6.2 also offers better signal quality compared to single link transmission. SNRC and ESNRC show roughly the same performance. ERC and FRC intersect at SNR 14dB. After the cross-sectional point FRC offers lower BER.

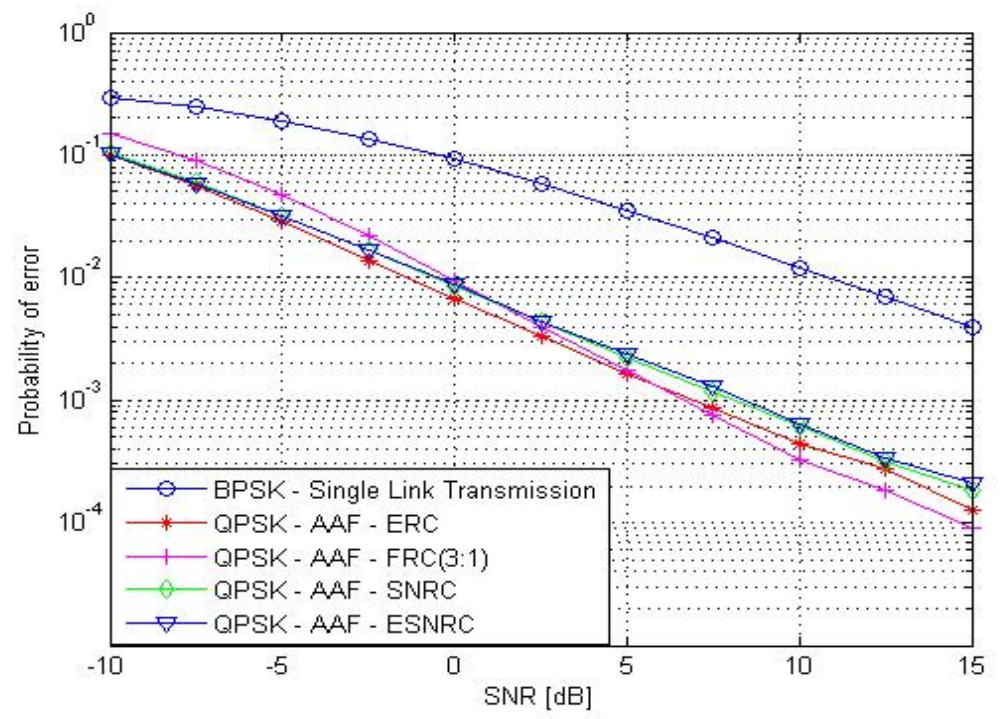

Fig 6. 1: - The different combining types using AAF for two-relay system are compared

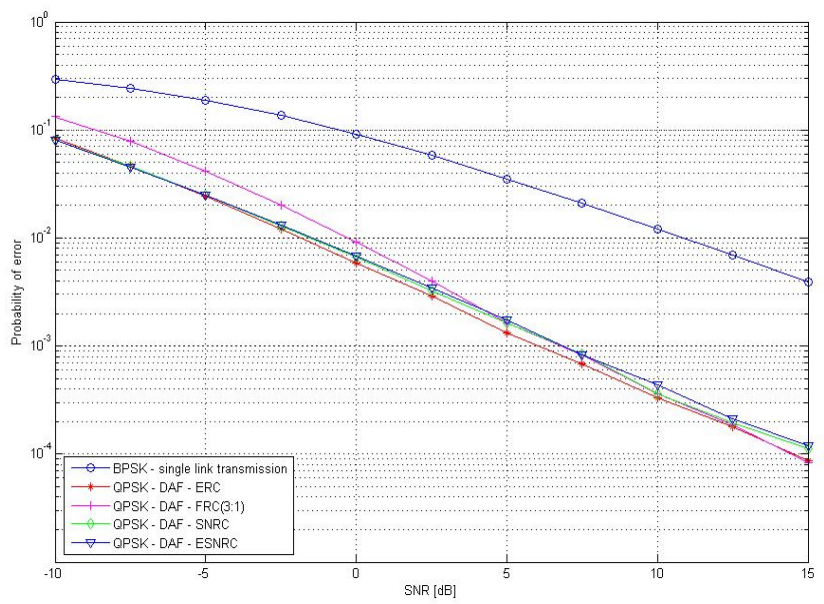

Fig 6. 2: - Different combining types using DAF for two relay system are compared with each other.

\section{Performance analysis between single relay and two relay cooperative systems}

The performances for single relay and two relay cooperative systems are analyzed from Figs. 6.3 to 6.6. The overall comparison for both AAF and DAF diversity protocols are represented in the followings 


\section{Amplify and forward (AAF)}

In Figure 6.3, the BER versus SNR curves for ERC and FRC are represented where both single relay and two relay cooperative systems use AAF retransmission protocol. In two relay cooperative system both ERC and FRC offer lower BER than corresponding single relay ERC and FRC

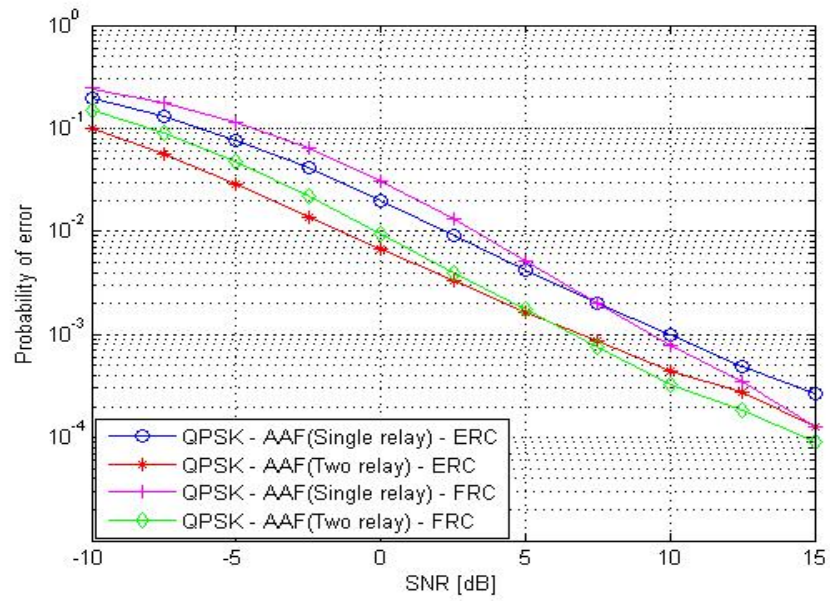

Fig 6. 3: - Comparison of the single relay and two relay cooperative systems using AAF for ERC and FRC.

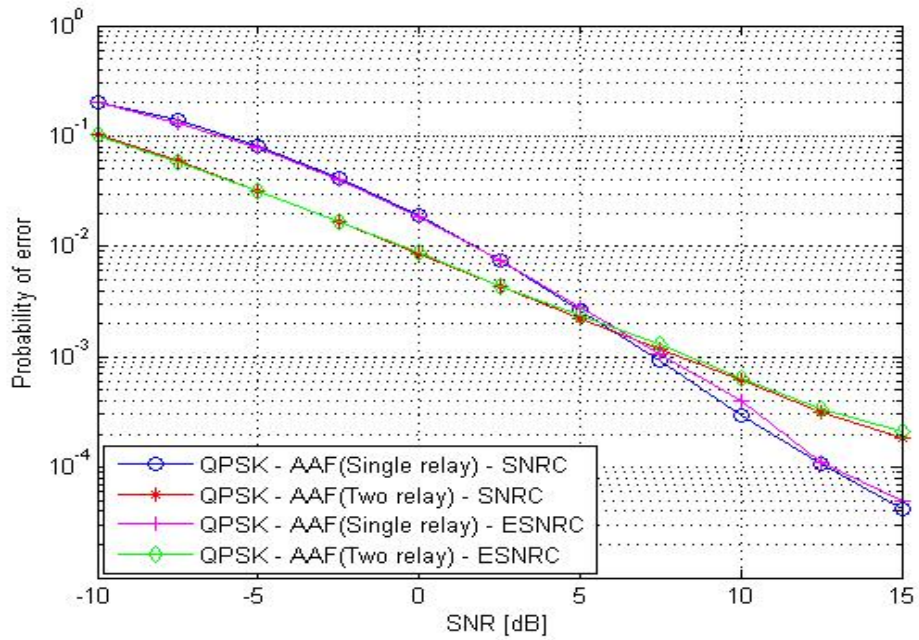

Fig 6. 6: - Comparison of the single relay and two relay cooperative systems using AAF for SNRC and ESNRC.

In Figure 6.4, the BER versus SNR curves for SNRC and ESNRC are represented where both single relay and two relay cooperative systems use AAF retransmission protocol. For single relay and two relay cooperative systems SNRC shows same probability of error (BER) $10^{-2.77}$ at $6.06 \mathrm{~dB}$. Two relay cooperative system provides improved performance when the SNR is lower than $6.06 \mathrm{~dB}$ but after that value of SNR single relay cooperative system shows better performance. ESNRC curves for both single relay and two relay intersect at $6.14 \mathrm{~dB}$ where BER is $10^{-2.75}$. The performance of single relay system is clearly improved when the SNR is greater than 6.14dB.

\section{Decode and forward (DAF)}

From the simulation curve of Figs. 5.5 and 5.6, it is clearly identified that, lower BER is achieved for two relay cooperative system compared to the single relay cooperative system. 


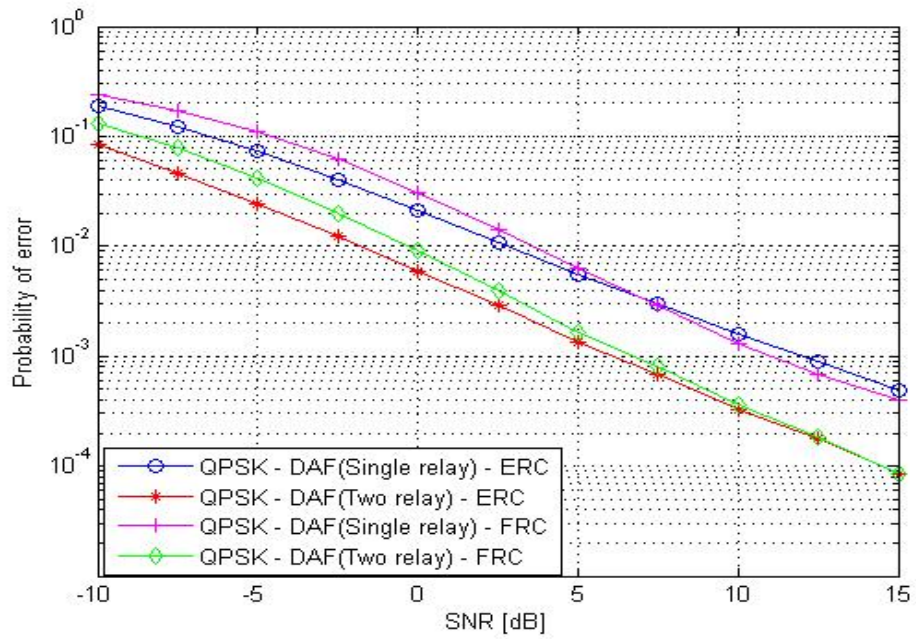

Fig 6. 5: - Comparison of the single relay and two relay cooperative systems using DAF for ERC and FRC.

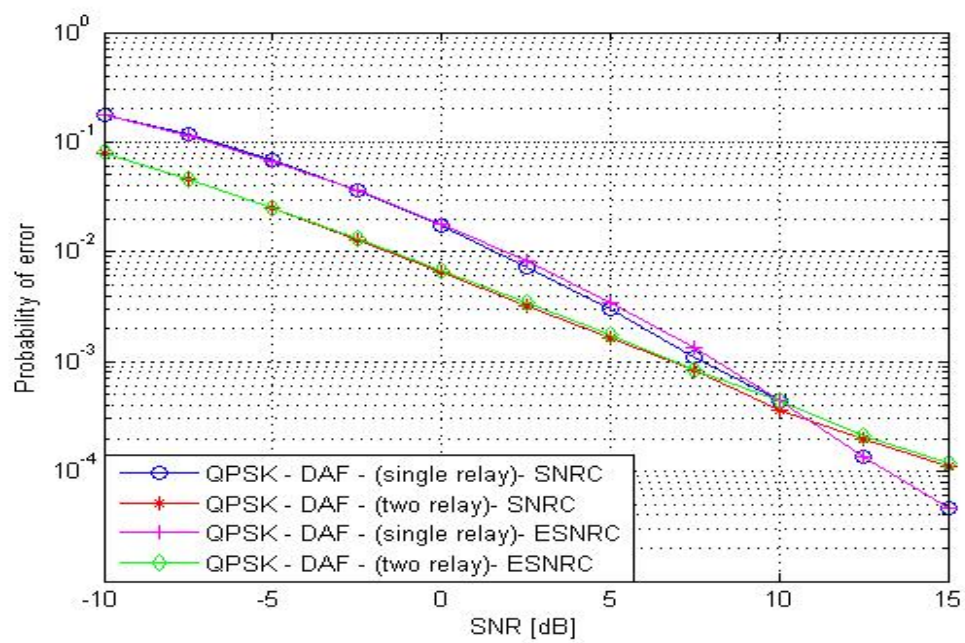

Fig 6. 6: - Comparison of the single relay and two relay cooperative systems using DAF for SNRC and ESNRC.

Two relay cooperative system always results in the better performance. BER $10^{-3.53}$ is achieved at $10.86 \mathrm{~dB}$ for both single relay and two relay cooperative system when SNRC combining technique is used at the destination. Though, two relay cooperative system offers better performance for SNRs lower than $10.86 \mathrm{~dB}$ but the performance of single relay system is improved after $10.86 \mathrm{~dB}$. From the cross-sectional point (SNR $10.06 \mathrm{~dB}$, BER $10^{-3.37}$ ) the performance of single relay system is better at higher SNRs for ESNRC.

\section{Conclusion: -}

In the comparative study conducted on the performance of single relay and two relay cooperative systems it was observed that, when ERC and FRC have been implemented for both AAF and DAF diversity protocols then much better system performance has been offered by the two-relay cooperative system compared to the single relay cooperative system. For SNRC and ESNRC two relay cooperative system has shown improved system performance at lower SNRs.

\section{References: -}

1. F. A. Tobagi, "Modeling and performance analysis of multihop packet radio networks," Proc. IEEE, 75, 1987 (135-155).

2. John Boyer David D. Falconer and Halim Yanikomeroglu, Multihop Diversity in Wireless Relaying Channels, IEEE Transaction on Communications Vol. 52(10), (2004) 1820-1830. 
3. A. Mahmood, Cooperative Diversity in Wireless Networks, Journal of Engineering Science and Technology Review 3 (1) (2010) 184-187.

4. Rodoplu V and Meng TH. "Minimum energy mobile wireless networks" IEEE Journal on Selected Areas in Communications, 17(8) (1999) 1333 - 1344.

5. Boyer J., Falconer D. and Yanikomerogl H. 2001 "A theoretical characterization of the multi-hop wireless communications channel with diversity," In Proceedings of IEEE Global Telecommunications Conference, 2 (2001) 841-845.

6. Nemanja Zdravkovic, Aleksandra Cvetkovic, Kimmo Kansanen and Goran T. Djordjevic, Outage performance of low-latency decode-and-forward cooperative wireless networks, EURASIP Journal on Wireless Communications and Networking, 95 (2016) 3-10.

7. Ahmed K. Sadek, Weifeng Su and K. J. Ray Liu, Multinode Cooperative Communications in Wireless Networks, IEEE Transaction on Signal Processing Vol. 55(1), (2007) 341-355.

8. Boyer J., Falconer D. and Yanikomeroglu "A theoretical characterization of the multi-hop wireless communications channel without diversity," In Proceedings of IEEE International Symposium on Personal, Indoor and Mobile Radio Communications 2, (2001)116-120.

9. Laneman JN, Tse DNC and Wornell GW "Cooperative Diversity in Wireless Networks: Efficient Protocols and Outage Behaviour," 50, (2004) 3062-3080.

10. Veluppillai Mahinthan, Lin Cai, Jon W. Mark, and Xuemin (Sherman) Shen, Maximizing Cooperative Diversity Energy Gain for Wireless Networks, IEEE Transaction on Wireless Communications, 6(6) (2007) 2530 - 2539.

11. Mohammadali Mohammadi* , Mehrdad Ardebilipour, Zahra Mobini and Ramezan-Ali Sadegh Zadeh, Performance analysis and power allocation for multi-hop multi-branch amplify-and-forward cooperative networks over generalized fading channels, EURASIP Journal on Wireless Communications and Networking, 160 (2013) 2-13.

12. Nosratinia A, Hunter TE, Hedayat A, “Cooperative Communication in Wireless Networks"IEEE Communication Magazine (2004) 74-80.

13. Yuksel M and Erkip E. "Diversity in relaying protocols with Amplify andforward," Polytechnic University. Brooklyn, New York, 4 (2003)2025-2029.

14. Ahmed S Ibrahim Ahmed K. Sadek Weifeng Su K. J. Ray Liu, “Cooperative Relaying”, 7, (2008) 2814-2827.

15. V. Mahinthan, J. W. Mark, and X. Shen, "A cooperative diversity scheme based on quadrature signaling," IEEE Trans. Wireless Commun., 6(1) (2007) 41-45.

16. J. H. Winters, "The diversity gain of transmit diversity in wireless systems with Rayleigh fading," IEEE Trans. Veh. Tech., 47(1) (1998) 119-123.

17. E. Zimmermann, P. Herhold, and G. Fettweis, "A novel protocol for cooperative diversity in wireless networks," in Proc. Fifth European Wireless Conference Mobile and Wireless System Beyond 3G (2004) 20-26.

18. Z. Wang and G. B. Giannakis, "A simple and general parameterization quantifying perfromance in fading channels,” IEEE Trans. Commun., 51 (2003), 1389-1398.

19. Meier A, and Thompson JS "Cooperative Diversity in Wireless Network" Emerging technology conference proceeding (2010) 359-363.

20. Mingwei CAO, Guangguo BI, Xiufeng JIN, Bandwidth Efficient Three-User CooperativeDiversity Scheme Based on Relaying Superposition Symbols, Wireless Sensor Network,1 (2009)1-60. 